\title{
ON SUPERSOLUBILITY IN SOME GROUPS WITH FINITELY GENERATED FITTING RADICAL
}

\author{
JAMES C. BEIDLEMAN AND HOWARD SMITH
}

(Communicated by Warren J. Wong)

\begin{abstract}
The groups $G$ considered in this paper have the property that every normal nonsupersoluble subgroup of $G$ has a finite, $G$-invariant, nonsupersoluble image. The structure of a certain radical, defined in terms of ascendant supersoluble subgroups, is determined. The main result is a supersolubility criterion for polycyclic groups.
\end{abstract}

\section{INTRODUCTION}

A theorem due to Baer ([3] or [15, p. 162]) states that a polycyclic group $G$ is supersoluble if and only if every finite image of $G$ is supersoluble. In [4] the associated property $\sigma$ was considered: a group $G$ has property $\sigma$ if every normal nonsupersoluble subgroup $N$ of $G$ contains a $G$-invariant subgroup $L$ such that $N / L$ is finite and nonsupersoluble. Free groups and subgroups of $\operatorname{GL}(n, \mathbb{Z})$ satisfy $\sigma$. Using a result of Huppert [8] we note that a group $G$ with $\sigma$ is supersoluble if and only if every maximal subgroup of $G$ has prime index. [4, Proposition 1] is used here to deduce that, in a group with $\sigma$, the subgroup generated by all ascendant supersoluble subgroups is a finite extension of a finitely-generated nilpotent group (Theorem 6). Our main result, however, is the following, which generalizes a theorem of Kramer ([9] or [16, Chapter 1, Theorem 3.3]) on finite soluble groups. Here, as elsewhere, Fit $G$ denotes the Fitting radical of the group $G$.

Theorem 1. Let $G$ be a polycyclic group. Then $G$ is supersoluble if and only if for every maximal subgroup $M$ of $G$, either Fit $G$ is contained in $M$ or $M \cap$ Fit $G$ is a maximal subgroup of Fit $G$.

One might attempt to extend Theorem 1 to include other classes of groups with $\sigma$. However, from [4] we know that a soluble group with $\sigma$ is polycyclic and so there is no improvement possible along these lines. Further, "polycyclicby-finite" is not going to be sufficient, since any finite nonabelian simple group trivially satisfies the stated condition on maximal subgroups. We recall also (see

Received by the editors June 27, 1990.

1980 Mathematics Subject Classification (1985 Revision). Primary 20F16, 20E28; Secondary 20E15, $20 \mathrm{E} 26$.

The second author acknowledges the kind hospitality of the Department of Mathematics at the University of Kentucky. 
[4] or [12, 53]) that McLain's insoluble, locally soluble group with the maximal condition on normal subgroups satisfies $\sigma$ and has trivial Fitting subgroup.

\section{THE PROOF OF THEOREM 1}

Suppose $G$ is a polycyclic group. The necessity of the condition on maximal subgroups is easily established, for if $G$ is supersoluble then every maximal subgroup of $G$ has prime index in $G[13,5.4$.7] and hence contains a subgroup of prime index in Fit $G$. For the converse, suppose that every maximal subgroup of $G$ contains a maximal subgroup of $F=$ Fit $G$, and let $\Phi(G)$ denote the Frattini subgroup of $G$. Then $\Phi(G)$ is of course the intersection of all maximal subgroups of $G$ and each of these has finite index in $G$, a result which is due to P. Hall [6]. From [4, Theorem 4] we have that $G$ is supersoluble if and only if $G / \Phi(G)$ is supersoluble. (This is also a special case of a theorem of Lennox [10].) Further, we know from another result of Hall [7] that $\Phi(G) \leq$ Fit $G$ and Fit $(G / \Phi(G))=($ Fit $G) / \Phi(G)$. Taken together, these results allow us to factor by $\Phi(G)$, and from now on we assume that $\Phi(G)=1$.

Before proceeding, we introduce the following lemma, which will soon be required and whose proof is deferred until the end of this section.

Lemma 2. Let $T$ be a finite, nontrivial, abelian normal subgroup of the group $G$ and suppose that $G$ has trivial Frattini subgroup. Then $T$ is a direct product of minimal normal subgroups of $G$.

The remainder of the proof of Theorem 1 is broken up into a series of steps. Recall that $F=$ Fit $G$, which is finitely generated and abelian (see [15, Lemma 4.15]).

(1) It suffices to prove that, for some set $\left\{N_{\lambda}: \lambda \in \Lambda\right\}$ of $G$-invariant subgroups of finite index in $F$ such that $\bigcap_{\lambda \in \Lambda} N_{\lambda}=1,\left[G^{\prime}, F\right] \leq N_{\lambda}$, for all $\lambda$ :

Assuming there is such a set of subgroups, it is clear that $G^{\prime}$ lies in the centralizer of $F$ and hence in $F$. Let $M$ be maximal in $G$. If $F$ is contained in $M$ then $G^{\prime} \leq M$ and so $M$ has prime index in $G$. Otherwise, by hypothesis, $|F: F \cap M|$ is prime and hence $|F M: F|=|G: M|$ is again prime. It follows ([8] or $[13,9.5 .4])$ that every finite image of $G$ is supersoluble and hence that $G$ is supersoluble by Baer's Theorem ([3] or [15, p. 162]).

(2) If $N$ is a $G$-invariant subgroup of $F$ such that $F / N$ is finite and $\Phi(G / N)=1$ then $\left[G^{\prime}, F\right] \leq N$ :

In this case, Lemma 2 can be applied to deduce that $F / N$ is a direct product of minimal normal subgroups of $G / N$. Let $P / N$ be one such and let bars denote factor groups modulo $N$. By hypothesis, there is a maximal subgroup $\bar{M}$ of $\bar{G}$ such that $\bar{P} \not \bar{M}$. Hence $\overline{M P}=\bar{G}$ and $\bar{M} \cap \bar{P}$ is a normal subgroup of $\bar{G}$ which does not contain $\bar{P}$ and is therefore trivial. Now $M$ does not contain $F$ and so $|F: F \cap M|=p$, a prime. It follows easily that $|\bar{P}|=p$. $\bar{G} / C_{\bar{G}}(\bar{P})$ is then abelian, and, since this is the case for all such subgroups $\bar{P}$, we get $\left[G^{\prime}, F\right] \leq N$, as required.

Next, we introduce some further notation and some results that will simplify the remainder of the proof. Let $L$ denote the intersection of all maximal subgroups of $G$ containing $F$ and choose a set of representatives $\left\{M_{\lambda}: \lambda \in \Lambda\right.$ \} of the conjugacy classes of those maximal subgroups of $G$ that do not contain $F$. For each $\lambda$ denote by $C_{\lambda}$ the normal core of $M_{\lambda}$ in $G$. Then $G / C_{\lambda}$ is 
finite and $L / F=\Phi(G / F)$. Further, by a well-known theorem of Mal'cev ([11] or $[12$, pp. 79,80$]) G / F$ is abelian-by-finite and, of course, finitely generated. Once again we appeal to a result of P. Hall [6, Lemma 10] to conclude that $L / F$ is finite. We are now ready for the final two steps.

(3) It suffices to prove that, for some $\lambda_{1}, \ldots, \lambda_{r}, C \cap L \leq F$, where $C=$ $C_{\lambda_{1}} \cap \cdots \cap C_{\lambda_{r}}$ :

For suppose this is so and, for each $\lambda \in \Lambda$, let $N_{\lambda}=C \cap L \cap C_{\lambda}$, a $G$-invariant subgroup of finite index in $F$. Then $\cap N_{\lambda} \leq \bigcap C_{\lambda} \cap L=\Phi(G)=1$ (where the intersections are over all $\lambda \in \Lambda$ ). Also, each $N_{\lambda}$ is an intersection of maximal subgroups of $G$ and so $\Phi\left(G / N_{\lambda}\right)=1$. Steps (1) and (2) now give the result.

(4) The construction of the subgroup $C$ :

Suppose $A / F$ is a normal subgroup of $G / F$ such that $F<A \leq L$. (If none such exists then $F=L$ and any $C_{\lambda}$ will do for $C$-indeed the construction of $C$ becomes unnecessary.) Then there exists $C_{\lambda}$ such that $F\left(A \cap C_{\lambda}\right) \neq A$, otherwise $A^{\prime} \leq A \cap C_{\lambda}$ for all $\lambda$ and $A^{\prime} \leq L$, giving $A^{\prime}=1$, in contradiction to the definition of $F$. Applying this argument repeatedly to an appropriate sequence of subgroups of the finite group $L / F$, we obtain the desired sequence $\left\{C_{\lambda_{i}}\right\}$. This concludes the proof of Theorem 1 .

Proof of Lemma 2. With the hypotheses of the lemma satisfied, let $N$ be the product of all minimal normal subgroups of $G$ contained in $T$. It suffices to prove that $N=T$. Certainly $N$ is nontrivial and so there is a maximal subgroup $M$ of $G$ such that $G=M N$. Choose $H$ of largest index in $G$ subject to $G=H N$. Then $H \cap N$ is normal in $G$, and we proceed to show that $H \cap N=1$. If this is not the case then there is a maximal subgroup $L$ of $G$ such that $G=(H \cap N) L$, thus implying that $H=(H \cap N)(H \cap L)$ and hence that $H \cap N \not \leq \Phi(H)$ (else $H=H \cap L$, a contradiction). But this in turn means that there is a maximal subgroup $W$ of $H$ such that $H=W(H \cap N)$, giving $G=W N$, in contradiction to the choice of $H$. Thus $H \cap N=1$. Now $H \cap T$ is normal in $G=H T$ and, since $H \cap T \cap N=1$, it follows that $H \cap T$ contains no minimal normal subgroup of $G$ and therefore is trivial. Therefore $T=T \cap H N=(T \cap H) N=N$, as required.

It may be worth remarking that the construction in Step 4 of the proof of Theorem 1 is not dependent on the maximal subgroup property concerned. In fact, it is not difficult to see that the following holds.

Proposition 3. Let $G$ be a polycyclic-by-finite group and denote by $F$ the Fitting radical of $G$. Suppose $\Phi(G)=1$. Then there exists a set $\left\{N_{\lambda}: \lambda \in \Lambda\right\}$ of $G$ invariant subgroups of $F$ such that

(i) $F / N_{\lambda}$ is finite and $\Phi\left(G / N_{\lambda}\right)=1$ for all $\lambda \in \Lambda$ and

(ii) $\bigcap_{\lambda \in \Lambda} N_{\lambda}=1$.

\section{ASCENDANT SUPERSOLUBLE SUBGROUPS OF GROUPS WITH PROPERTY $\sigma$}

As suggested in the introduction, the result that provides a starting-point for our discussion in this final section is [4, Proposition 1], where it is proved that the iterated Hirsch-Plotkin radical $H(G)$, say, of a group $G$ with $\sigma$ is polycyclic. Now in any group $G$ the (characteristic) subgroup generated by all ascendant soluble subgroups is contained in $H(G)$-this may be seen by first 
considering the subgroup generated by all ascendant abelian subgroups, which is normal and contained in the Hirsch-Plotkin radical of $G$ ([5] or [12, p. 59]), and proceeding inductively. Thus we have the following results.

Theorem 4. If $G$ has $\sigma$, then the subgroup $S$ of $G$ generated by all ascendant soluble subgroups is polycyclic.

Corollary 5. If $G$ has $\sigma$, then any ascendant soluble subgroup of $G$ is subnormal.

The corollary is immediate, since polycyclic groups satisfy the maximal condition. Note that "soluble" cannot be replaced by "locally soluble" or even "locally polycyclic" in the theorem (or its corollary) on account of McLain's group mentioned earlier.

If we restrict attention to supersoluble subgroups, we obtain a good description of the corresponding radical.

Theorem 6. If $G$ has $\sigma$, then the subgroup $S^{*}$ generated by all ascendant supersoluble subgroups of $G$ is (polycyclic and) nilpotent-by-(finite nilpotent).

Proof. Since $S^{*}$ is polycyclic, it is generated by finitely many subnormal supersoluble subgroups $S_{1}, \ldots, S_{n}$, say. By Proposition 7 below, we may assume that each $S_{i}$ contains the Fitting subgroup $F$ of $G$, which of course equals the Hirsch-Plotkin radical. Thus, for each $i=1, \ldots, n, F$ is contained in $F_{i}=$ Fit $S_{i}$. But $F_{i}$ is itself contained in the Baer radical of $G$ and hence equals $F$. Further, each $S_{i} / F$ is finite and abelian and so, modulo $F, S^{*}$ is nilpotent. (These facts concerning subgroups generated by subnormal nilpotent subgroups are of course due to Baer ([1] or [12, 2.3]).) The following proposition, therefore, completes the proof of Theorem 6.

Proposition 7. Suppose $K$ is a subnormal supersoluble subgroup and $L$ is a finitely generated, normal nilpotent subgroup of the group $J$ which is generated by $K$ and $L$. Then $J$ is supersoluble.

Proof. With the hypotheses as stated, $J$ is certainly polycyclic. Suppose, for a contradiction, that $J$ is not supersoluble. Then, by ([3] or [15, p. 162]), $J$ has a finite nonsupersoluble image, and we may as well assume that $J$ is finite. Since $K^{J}=K\left(K^{J} \cap L\right)$, we may further assume, by induction on the order of $J$, that $K$ is normal in $J$. By another theorem of Baer ([2] or [16, Chapter 1, Theorem 3.3]), it suffices to prove that $J^{\prime}$ is nilpotent. But $J^{\prime}$ is a subgroup of $K^{\prime} L$, a product of two normal nilpotent subgroups. The desired contradiction follows from Fitting's Theorem.

The above result extends in a routine way:

Corollary 8. Suppose $K$ and $L$ are subnormal subgroups of $J=\langle L, K\rangle$ and suppose further that $K$ is supersoluble and $L$ is finitely generated nilpotent. Then $J$ is supersoluble.

Proof. The subgroup $L^{J}$ is generated by conjugates of $L$ and hence by finitely many such, by the maximal condition. $L^{J}$ is then nilpotent [1] and Proposition 7 applies.

We mention here the well-known example of Huppert [14, p. 219] which shows that a product $G$ of normal supersoluble subgroups need not be supersoluble, even if $G$ is finite. In fact, the theorem of Baer referred to in the proof 
of Proposition 7 states that such a product $G$ is supersoluble if and only if $G^{\prime}$ is nilpotent, and the nilpotency of the derived subgroup is one of the hypotheses in our final (and related) result, which has an obvious application to the radical $S^{*}$ defined in Theorem 6 .

Proposition 9. Let $G$ be a group which is generated by finitely many ascendant supersoluble subgroups $H_{1}, \ldots, H_{n}$. Then $G$ is supersoluble if and only if $G^{\prime}$ is nilpotent.

Proof. The implication in one direction is well known. So suppose that $G$ is generated by such subgroups and that $G^{\prime}$ is nilpotent. Then $G$ is polycyclic (see [12, Theorem 2.33]) and it suffices to prove that all finite images of $G$ are supersoluble. We may thus assume that $G$ is finite. Then joins of subnormals are again subnormal, by a theorem of Wielandt [17], and so, by induction, we may further assume that $G=\langle H, K\rangle$, where $H$ and $K$ are subnormal and supersoluble. The proof is completed by means of a standard argument, using induction on the order of $G$ to reduce to the case where $H$ and $K$ are normal and then applying Baer's theorem ([2] or [16, Chapter 1, Theorem 3.3]).

Remark. With reference to Theorem 1, a remark by a referee has prompted us to say a few words concerning finitely generated soluble groups. It appears that there is not much hope of extending this result to such groups in any obvious way. The group $G$ with presentation $\left\langle x, y: x^{y}=x^{2}\right\rangle$ is metabelian of rank 2 (being an extension of the (additive) group of dyadic rationals by an infinite cyclic group). It is easily shown that every maximal subgroup of $G$ has prime index in $G$ and so $G$ satisfies appropriate hypothesis on maximal subgroups. However, $G$ is not polycyclic.

\section{REFERENCES}

1. R. Baer, Nilgruppen, Math. Z. 62 (1955), 402-437.

2. __ Classes of finite groups and their properties, Illinois J. Math. 1 (1957), 115-187.

3. __ Überauflösbare Gruppen, Abh. Math. Sem. Univ. Hamburg 23 (1957), 11-28.

4. J. C. Beidleman and D. J. S. Robinson, On the structure of the normal subgroups of a group: Supersolubility, Rend. Sem. Mat. Univ. Padova (to appear).

5. K. W. Gruenberg, The Engel elements of a soluble group, Illinois J. Math. 3 (1959), 151-168.

6. P. Hall, On the finiteness of certain soluble groups, Proc. London Math. Soc. (3) 9 (1959), 595-622.

7. (1961), 327-352.

8. B. Huppert, Normalteiler und maximale Untergruppen endlicher Gruppen, Math. Z. 60 (1954), 409-434.

9. O. U. Kramer, Über Durchschnitte von Untergruppen endlicher auflösbarer Gruppen, Math. Z. 148 (1976), 89-97.

10. J. C. Lennox, A supersolubility criterion for finitely generated hyper-(abelian-by-finite) groups, Arch. Math. 24 (1973), 247-248.

11. A. I. Mal'cev, On certain classes of infinite soluble groups, Amer. Math. Soc. Transl. 2 (1956), 1-21.

12. D. J. S. Robinson, Finiteness conditions and generalised soluble groups, vol. 1, SpringerVerlag, Berlin, 1972.

13. _ A course in the theory of groups, Springer-Verlag, New York, 1982.

14. W. R. Scott, Group theory, Prentice-Hall, New Jersey, 1964. 
15. B. A. F. Wehrfritz, Infinite linear groups, Springer-Verlag, Berlin, 1973.

16. M. Weinstein, Between nilpotent and solvable, Polygonal Publ. House, 1982.

17. H. Wielandt, Eine Verallgemeinerung der invarianten Untergruppen, Math. Z. 45 (1939), 209-244.

Department of Mathematics, University of Kentucky, Lexington, Kentucky 40506

Department of Mathematics, Bucknell University, Lewisburg, Pennsylvania 17837 